\title{
A fundamental study of parameter adjustable additive manufacturing process based on FDM process
}

\author{
Qia Wan*, Youjian Xu, and Can Lu \\ Department of Mechanical and Aerospace Engineering, University of Florida, Gainesville, FL 32611, \\ U.S.A.
}

\begin{abstract}
In Fused deposition modeling (FDM) process, there has been a confliction between high productivity and high quality of products. The product resolution is proportional to the flow rate of heated material extrusion, which directly affects the build time. To reduce the build time with acceptable resolution, the idea of parameter adjustable printing process has been introduced. The controllable extruder was modified and two types of diameter changeable nozzle have been designed. This work realizes different resolution building based on the part geometry during FDM process, which can efficiently assure the quality of products and improve the productivity at the same time.
\end{abstract}

\section{Introduction}

Additive manufacturing (AM) process has been widely known for its flexibility by fabricating complex geometric shapes in a fairly timeframe. Fused deposition modeling (FDM), as known in figure 1, also called material extrusion, is one of the additive manufacturing techniques that accomplishes the part by depositing the heated material layer by layer drawn through a nozzle. The rollers in the extruder generate the pressure to squeeze the material into the liquefier, then the material is melted into liquid or semi-liquid form and exits from the nozzle with $0.3 \mathrm{~mm}$ to $1.0 \mathrm{~mm}$ diameter. The heated material solidifies quickly and forms so-called filament once contacting with the platform or previous layer [1-3].

It is believed that small cross-sectional area of each material extrusion stand contributes high resolution of the part during the material extrusion processing. However, the smaller cross-sectional filaments lead slower material extrusion rate, which will directly increasing the build time. Several attempts have been proposed to solve this dilemma. K. Thrimurthulu et al. [4] obtained an optimum part deposition orientation for improving the surface finish and improving the build time. Tata et al. [5] applied the maximal permissive layer thickness for each layer to realize the higher productivity but constraint surface finish. Alexander and Dutta [6] reduced the build time by targeting shell-like structure with lower support volume. However, few attempts have been determined by adjusting extrusion parameters during FDM process in which the resolution can be controllable. In most cases, the resolution of

\footnotetext{
*Corresponding author: wqwqwqwq8526@ufl.edu
} 
the part is unnecessarily to be the same. High resolution is usually needed in surfaces with high slop rate since the stair-case effect will be less obvious in the surfaces with low slope rate than those with high slope rate [7], while relatively low resolution can be made in other surfaces. Therefore, adjusting the resolution during the printing process guarantees the appropriate surface finish with less build time. Nozzle diameter is one of the important factors for FDM process as it affects the resolution as well as material extrusion rate. Other aspects, such as pressure and temperature, are also needed to be determined [8]. Figure 2 shows the basic idea of the relationship between different factors. The needed resolution of different regions of the part should be determined based on the geometry of the desired part. Once the resolution has been known, the extrusion parameters that corresponding to the certain resolution can be calculated. Therefore, The relationship between the part geometry and extrusion parameters will be found.

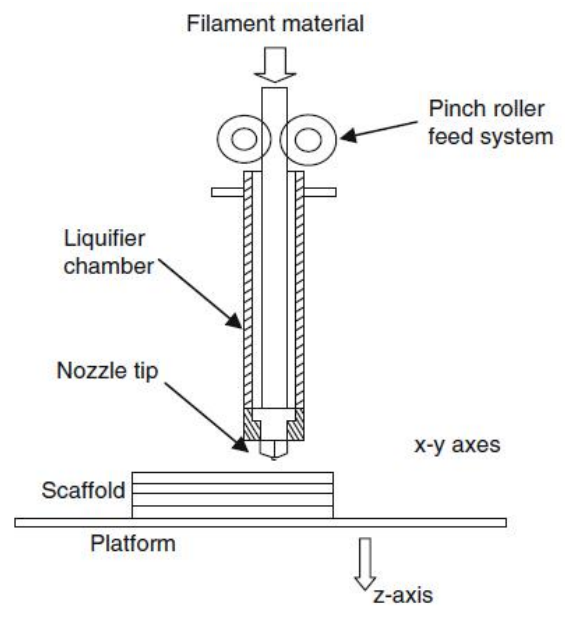

Fig. 1. Schematic of Fused deposition modelling [2].

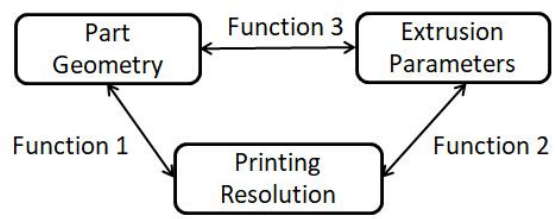

Fig. 2. Relationship between FDM factors .

In this paper, the nozzle diameter was chosen as the main changeable extrusion parameter. The extruder of the printer was modified to fit the new process, which determined the extrusion parameters under the certain resolution. The relationship between the part geometry and needed resolution was derived and two kinds of diameter changeable nozzle were designed for the process.

\section{Extruder modification}

Positive displacement gear pump is commonly used for precise metering of viscous fluid flows $[9,10]$. The flow rate is mainly governed by the rotation rate of the gears inside the pump. The effect of pressure and temperature in the pump can be neglected, which is suitable for the extrusion process. Therefore, in this paper, the gear pump was used in the 
extruder of the printer, and the rotation rate of the gears are considered as the only characteristic of flow rate from an ideal point of view.

\subsection{Pump flow analysis}

For ideal analysis, the following assumptions should been considered in the pump flow analysis [11]: (1) the fluid is incompressible, (2) fluid leakage is neglected, (3) the pump parts are rigid and inflexible. Based on those assumptions, the total entering volume of the material equals the total volume exiting the pump discharge chamber. Figure 3 shows the schematic diagram of the gear mesh between two teeth which can instantaneously control the volume of discharging. Two gears, driving gear 1 and driven gear 2, are shown in the pump. The entering and exiting infinitesimally small volume of each gear was represent as $d V_{\text {enter } 1,2}$ and $d V_{\text {exit } 1,2}$, respectively. The governing equation is yielded as:

$$
\begin{gathered}
d V t=\left(d V_{\text {enter }-}-d V_{\text {exit } 1}\right)+\left(d V_{\text {enter } 1}-d V_{\text {exit } 1}\right) \\
d V_{\text {enter }_{1,2}}=\frac{1}{2} r_{a_{l, 2}}{ }^{2} d \theta_{1,2} \\
d V_{\text {exit }_{1,2}}=\frac{1}{2} \rho_{1,2} d \theta_{1,2} \\
d \theta_{2}=\frac{r_{p_{1}}}{r_{p_{2}}} d \theta_{1}
\end{gathered}
$$

where:

$r_{a_{1,2}}=$ addendum radius of gear 1 and gear $2, r_{p_{1}} r_{p_{2}}=$ pitch radius of gear 1 and gear

$2, \rho_{1,2}=$ contact radial dimension of gear 1 and gear 2 .

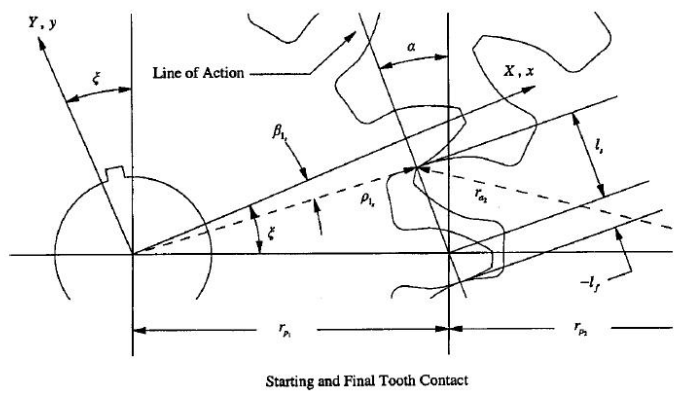

Fig. 3. Gear mesh geometry between two teeth [11].

substituting equations (2) - (4) into equation (1), and taking the derivative of time $d t$ to obtain the ideal instantaneous flow rate $Q$ of the gear pump:

$$
Q=\frac{d V_{t}}{d t}=\frac{1}{2}\left\{\left(\mathrm{r}_{\mathrm{a}_{1}}^{2}-\rho_{1}^{2}\right)+\frac{r_{p_{1}}}{r_{p_{2}}}\left(\mathrm{r}_{\mathrm{a}_{2}}{ }^{2}-\rho_{2}^{2}\right)\right\}
$$

\subsection{Extrusion filament analysis}

Based on the flow rate calculated in section 2.1, the mean extrusion speed can be obtained as:

$$
V=\frac{4 Q}{\pi d_{n o z z l e}{ }^{2}}
$$

where $d_{\text {nozzle }}$ is the nozzle diameter. Since the heated material extruded from nozzle piles up on the platform, the cross-sectional shape and area of filament are theoretically considered as the same as those of nozzle. The diameter of the filament can be represented as: 


$$
d_{\text {filament }}=d_{\text {nozzle }}=\left(\frac{4 Q}{\pi v}\right)^{\frac{1}{2}}=\left(\frac{2}{\pi v}\left\{\left(r_{a_{1}}^{2}-\rho_{1}{ }^{2}\right)+\frac{r_{p_{1}}}{r_{p_{2}}}\left(r_{a_{2}}{ }^{2}-\rho_{2}{ }^{2}\right)\right\}\right)^{\frac{1}{2}}
$$

During the extrusion, the mean extrusion speed can be set as the optimal one. The resolution is assumed to be determined only based on the filament diameter. Therefore, the desired flow rate is obtained by equation (7), which can be controlled by changing rotation rate. The speed of the nozzle movement is assumed to be the same as the material extrusion speed for a reliable resolution.

\section{Relationship between part geometry and needed resolution}

As being stated before, the needed resolution should be derived with respect to the part geometry, and the resolution can be represented only based on the filament diameter. The scope of the filament diameter is assumed to be the same as the scope of the extrusion nozzle diameter, which is nearly $0.1 \mathrm{~mm}$ to $0.5 \mathrm{~mm}$. Larger filament diameter may result in bad quality shape due to constraints of mechanical properties and surface finish [12]. In this paper, the filament diameter determination has been discussed only based on two criterions: external-slope criterion and small-feature criterion.

The external slope is mainly defined by the vertical boundaries of the geometry based on the building orientation. During the FDM process, filaments build each layer and form the corresponding vertical boundaries. The layer thickness should be equal to the filament diameter theoretically. However, the heated semi-solid filaments are compressed by adjacent layers in vertical direction, and then penetrate into each other. Therefore, the effective thickness of each layer, shown in figure 4, should be smaller than the filament diameter. Variable $\alpha$, the ratio of the filament effective thickness to the filament diameter, is involved in several factors, such as filament displacement, temperature, flow rate, pressure, etc. In this paper, the $\alpha$ has been treated as the constant for ideal calculation.

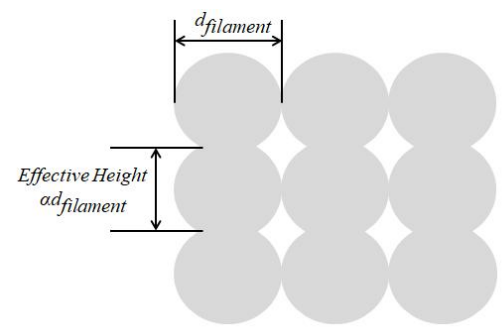

Fig. 4. Cross sectional shape of the extruded filaments.

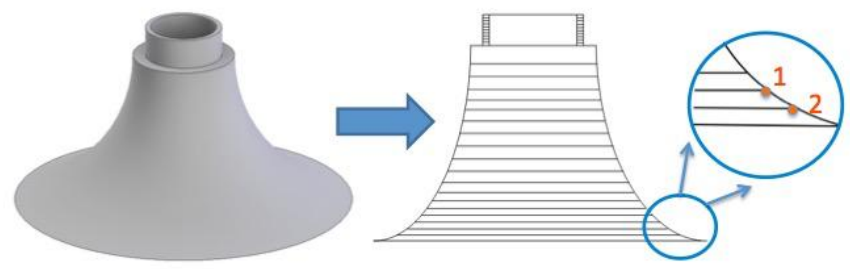

Fig. 5. The sliced part geometry with infinitesimal layers.

The external slope can be obtained by analyzing the slope of vertical boundaries of each layer of the part geometry. Specifically, the geometry is sliced into infinitesimal layers, shown in figure. 5. The coordinate of point 1 and point 2 on the boundary can be 
represented as $\left(x_{1}, z_{1}\right)$ and $\left(x_{2}, z_{2}\right)$, then the slope between the two points can be approximated as:

$$
K=\frac{z_{2}-z_{1}}{x_{2}-x_{1}}
$$

The slope should be converted to fit in the range of constraint filament diameter. Therefore, the relationship between the needed filament diameter $\mathrm{d}$ and the slope $\mathrm{k}$ of one of the vertical boundary of the sliced layers can be found as:

$$
d_{\text {filament }}=d_{\text {filament }_{\text {min }}}+\left(d_{\text {filament }_{\text {max }}}-d_{\text {filament }_{\text {min }}}\right) * \sin \left(\text { tan }^{-1}(|k|)\right)
$$

where $d_{\text {filament }_{\text {min }}}, d_{\text {filament }_{\text {max }}}$ is the minimum and maximum available diameter of the filament, respectively. The desired filament diameter should gradually increase as the boundary becomes steeper.

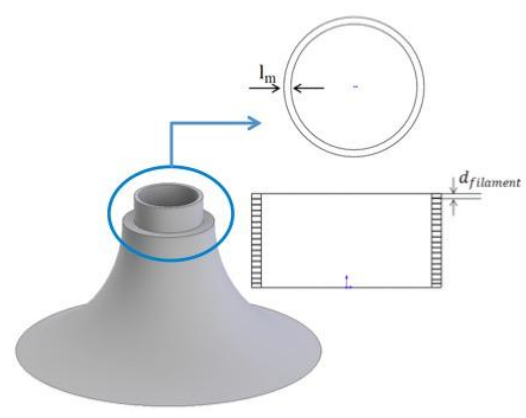

Fig. 6. The cylinder part with ring cross section in the part geometry.

The small feature criterion is mainly used for horizontal cross section building. Figure 6 shows the same example of small cylinder with ring cross section which may hardly be represented or fabricated by using the large-diameter filament based on the external-shape criterion. To build the ring cross sectional feature horizontally and follow the external slope criterion vertically (figure 5) at the same time, a basic diagram, shown as figure 7 , has been designed.

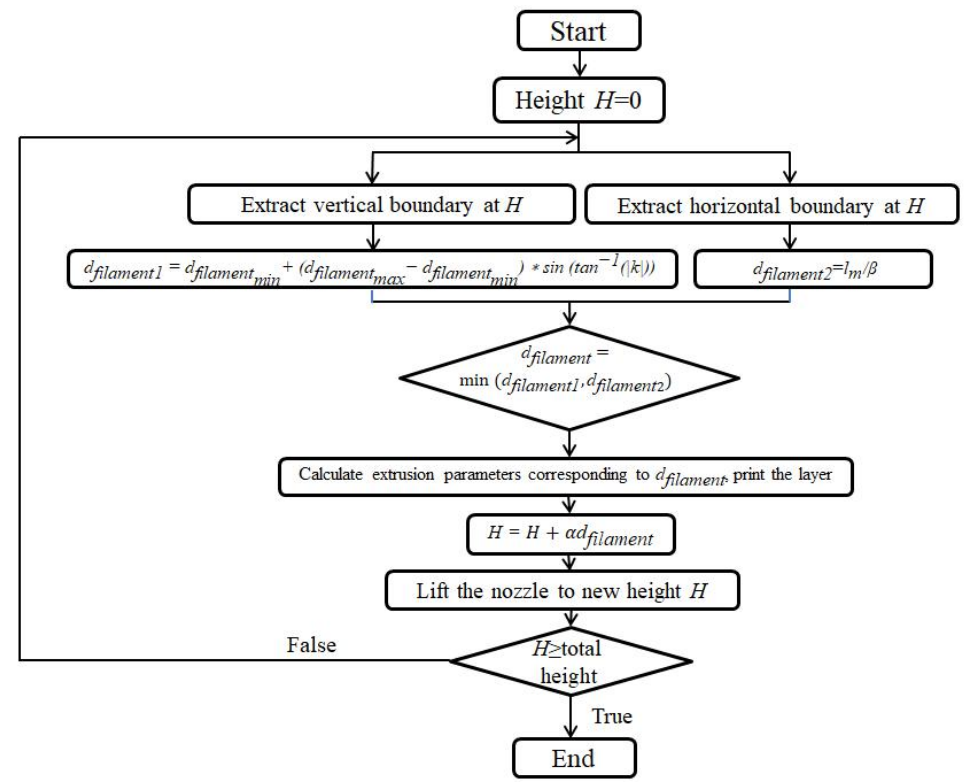

Fig.7. The algorithm diagram of parameter adjustable printing process. 
The minimum distance $\left(l_{m}\right)$ is obtained in the ring horizontal cross sectional feature. Then, the needed filament diameter of the portion can be calculated based on the minimum distance of the ring horizonal cross section:

$$
d_{\text {filament }}=l_{m} / \beta
$$

where coefficient $\beta$ represents the desired slicing number of the small feature. At the end the needed filament diameter calculated by small-feature criterion should be compared with the diameter calculated by external-slope criterion. The smaller one will be chosen for the layer.

The external-slope criterion reduces the stair-case effect and increases the productivity at the same, meanwhile the small-feature criterion allows a better fineness for small feature on the part. However, the two criterions are still remaining several issues. The penetration between the adjacent filaments horizontally in the same layer should be considered. Therefore, the effective width of the filament should be larger than the filament diameter, which may affect the resolution and shape quality on the internal sections. Moreover, either effective height or effective width of each filament varies during the building process due to the compression or deformations from the heat and pressure. It is hard to predict the single filament geometry without setting multiple assumptions.

\section{Design of the nozzle}

\subsection{Iris-diaphragm-shaped nozzle}

Iris diaphragm is an optical component that can feature a series of smooth, thin blades arranged to form a circular aperture that can be opened or closed depending on the application. It has been widely used in the shutter of the camera for limiting the amount of light transmitted to an imaging sensor or photo detector in order to prevent oversaturation. The main advantage of iris diaphragm is the controllable change of the aperture diameter. Therefore, it can be used as the nozzle component to realize the changeable nozzle diameter. Figure 8 shows the component of the Iris diaphragm, which usually consists of three parts: base, blades and turning disc. The blades are set up on the base, slid over each other in particular orientations, which may create a circular aperture. Usually, Iris diaphragm has two to eighteen blades, which the more blades comes the more accurate circular shape. The tuning disc uses arc channels to control the rotation of the blades, which can let the aperture stay open for the required time and dimension, and closes in the same way. In this project, a model of sixteen-curved-blade iris diaphragm nozzle has been designed, the maximum diameter of the nozzle is $0.5 \mathrm{~mm}$ and minimum diameter of the nozzle is $0.1 \mathrm{~m}$. Figure 9 shows the top view of the nozzle in maximum diameter and minimum diameter, respectively.

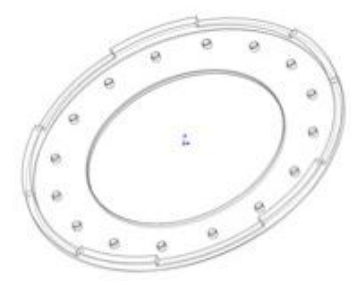

(A)

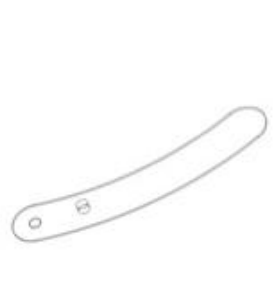

(B)

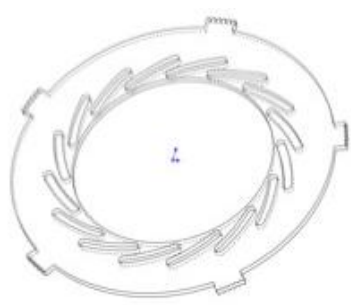

(C)

Fig. 8. Geometry of the parts of iris diaphragm (A)-base, (B)-curved blades, (C)-turning disc. 


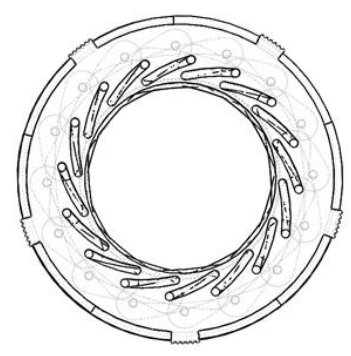

(A)

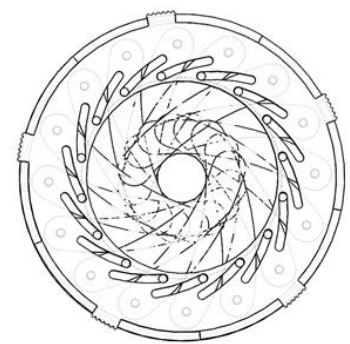

(B)

Fig. 9. Top view of the nozzle (A)-maximum diameter, (B)-minimum diameter.

Compared to the traditional extrusion printing nozzle, the iris-shaped nozzle can adjust the diameter easily and realize the changeable diameter during the printing process. The multi-blades of iris diaphragm can guarantee the circular cross-sectional shape of the nozzle. It is feasible to change the diameter of the nozzle precisely and rapidly by utilizing electronic control system. However, Even if the iris-shaped nozzle could realize the changeable diameter in 3D printing, it may have some defects as well. For instance, some gapes may exist around the circular aperture although the blades have been stacked on each other, it may cause the leaking problem during the extrusion. Besides, since the blades are extremely thin and small, the mechanical properties may be unsatisfied for perpendicular colliding of heated material. Moreover, during the extrusion, the blades may be softened due to the high temperature, which becomes harder to support the concentrated heated material and leads the catastrophic damages.

\subsection{Scroll nozzle}

Since the mechanical properties and assembly structure of the blades may cause the imprecision or failure during the printing process, it is necessary to carry out a new model without setting the extra planes in the nozzle. Then the scroll model has been designed originally to avoid those problems.

The inspiration of the model comes from the paper scroll, shown as Fig.10(A). Specifically, the paper can be somehow rolled as a cylinder scroll with maximum circular bottom shape, shown as Fig.10(B). Then, as the scroll being continually rolled, the circular bottom becomes smaller, shown as Fig.10(C) . And finally a spindly similar circulartruncated-cone scroll will be obtained with minimum circular bottom, shown as Fig.10(D) . The shape of the bottom can be kept as roundness all along. Therefore, the scroll model is likely to be designed as the diameter changeable nozzle for its easy diameter control.

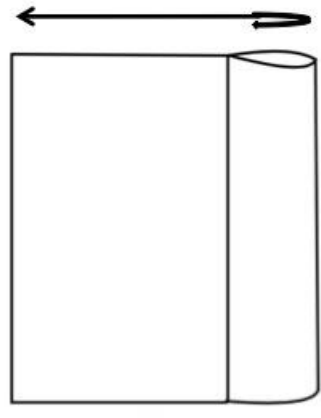

(A)

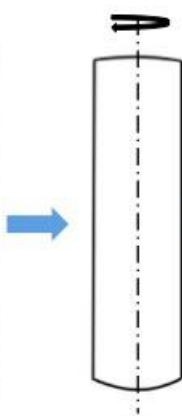

(B)

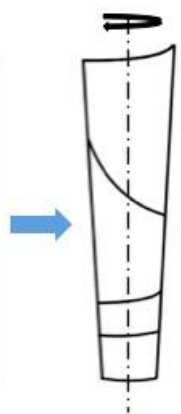

(C)

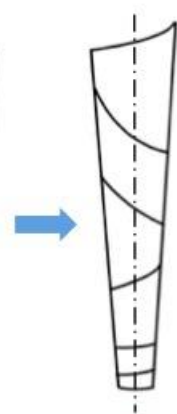

(D)

Fig. 10. Geometry of scroll nozzle. 
The plane structures are eliminated using scroll model so that the potential damages due to the plane failure or leaking problem can be avoid ideally. Moreover, the cross section of the scroll nozzle tip has been designed as strict roundness compared to the iris-diaphragmshaped nozzle, which may increase the precision during the extrusion. However, the scroll nozzle has been only proposed as row model. It is theoretically feasible but still remains plenty of challenges. For instance, the requirement of the scroll material is extremely harsh. Since the material needs to be rolled perfectly, just like paper, it should have great ductility and plasticity. Moreover, the material should have a good high temperature resistance in case of being melted during the extrusion in high temperature.

\section{Conclusion}

So far, the theoretical model for the parameter adjustable FDM process has been built up. The extruder of the printer was modified using positive displacement gear pump for controlling the flow rate by changing rotation rate so that the resolution, which is represented by filament diameter, could be adjusted by the flow rate during the extrusion process under certain optimal extrusion speed. The desired filament diameter of each building layer was determined by the part geometry using either external-slope criterion or small-feature criterion. Moreover, Two kinds of diameter changeable nozzle have been designed for the new process. However, several issues, such as the resolution and mechanical performances of the part internal sections or controlling and material selections challenges of the diameter changeable nozzle still remained to be solved for further study.

\section{References}

1. M.A. Yardimci, S. Güçeri, Rap.Prot.J. 2, 26-31 (1996)

2. M. Bernhard, Additive Manufacturing Technologies - Rapid Prototyping to Direct Digital Manufacturing (EGP, 2012)

3. B.V. Reddy, N.V. Reddy, A. Ghosh, Taylor \& Francis, 2, 51-60 (2007)

4. K. Thrimurthulu, P.M. Pandey, N.V. Reddy, Inter. J.of Mach. Tools and Mfg. 44, 585594 (2004)

5. ata, K., Fadel, G., Bagchi, A., Aziz, N. Prot. J. 4, 151-167 (1998)

6. P.Alexander, D.Dutta, C.A.D, 32, 175-189 (2000)

7. W. Oropallo, L.A. Piegl, Eng. With Comp. 22, 135-148 (2016)

8. Lu, X., Lee, Y., Yang, S., Hao, Y., Evans. J.R.G., Parini, C.G, J. of Matl. 209, 46544661 (2009)

9. Muth, J. T., Vogt, D. M., Truby, R. L., Mengüç, Y., Kolesky, D. B., \& Wood, R. J. Adv.Matl. 26, 6201-6353 (2014)

10. K. Saif, S. Wei, Matl. Sci. \& Eng. 28, 469-478 (2007)

11. M. Noah D, K. Suresh D, J. of Dynamic System. 125, 396-404 (2003)

12. T. Justin, B. Jan Helge, Matl.\& Desn, 20, 77-82 (1999) 